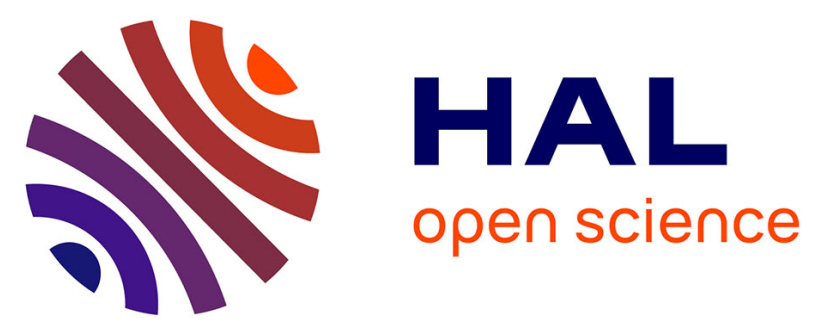

\title{
MICROANALYSE DES JOINTS \\ CARBURE-CARBURE DANS LES COMPOSITES CARBURE DE TUNGSTĖNE-COBALT
}

J. Laval, J. Vicens, G. Nouet

\section{- To cite this version:}

J. Laval, J. Vicens, G. Nouet. MICROANALYSE DES JOINTS CARBURE-CARBURE DANS LES COMPOSITES CARBURE DE TUNGSTĖNE-COBALT. Journal de Physique Colloques, 1984, 45 (C2), pp.C2-695-C2-700. 10.1051/jphyscol:19842161 . jpa-00223834

HAL Id: jpa-00223834

https://hal.science/jpa-00223834

Submitted on 1 Jan 1984

HAL is a multi-disciplinary open access archive for the deposit and dissemination of scientific research documents, whether they are published or not. The documents may come from teaching and research institutions in France or abroad, or from public or private research centers.
L'archive ouverte pluridisciplinaire HAL, est destinée au dépôt et à la diffusion de documents scientifiques de niveau recherche, publiés ou non, émanant des établissements d'enseignement et de recherche français ou étrangers, des laboratoires publics ou privés. 


\title{
MICROANALYSE DES JOINTS CARBURE-CARBURE DANS LES COMPOSITES CARBURE DE TUNGSTĖNE-COBALT
}

\author{
J.Y. Lava1, J. Vicens ${ }^{*}$ et G. Nouet ${ }^{*}$ \\ Laboratoire d'Etude et de Synthèse des Microstructures, ERA 912 , \\ CNRS-ESPCI, 10 rue Vauquelin, 75231 Paris Cedex 05, France \\ * Equipe Matériaux Microstmeture, Laboratoire de Cristallographie, Chimie \\ et Physique des Solides, IA 251, ISMRa, Université, 14032 caen Cedex, France
}

Résumé - La concentration en cobalt aux joints de grains carbure-carbure des composites WC-Co a été déterminée par microanalyse $\mathrm{X}$ à sêlection d'énergie en microscopie électronique à balayage en transmission. Il a été montré que seuls, les joints généraux se caractérisaient par une forte augmentation de la concentration en cobalt. I1 est possible d'en déduire la présence d'un squelette continu de carbure à travers les joints de coincidence.

Abstract - Cobalt concentration at WC-WC grain boundaries (G.B.) in WC-Co composites was determined via X-ray energy dispersive analysis in S.T.E.M. It was shown that only general G.B. were characterized by a high increase in cobalt. From these results, it was possible to infer a continuous carbide skeleton through coincidence G.B.

\section{INTRODUCTION}

Les matériaux composites WC-Co se caractérisent par la présence d'une phase dure (carbure de tungstène) noyée dans une phase ductile (cobalt). Cette microstructure granulaire confère à ce matériau des propriétés mécaniques remarquables en tant qu'outil de coupe pour les matériaux fragiles. La géométrie particulière des grains de carbure (prisme à base triangulaire) entraîne la formation de nomSreux joints plans carbure-carbure et de poches de cobalt. Cette morphologie définit un squelette continu ou quasi-continu de grains de carbure. Comme ce matériau est prêparé par frittage en phase liquide (cobalt), la prêsence de cobalt sous la forme d'une phase continue ou d'atomes ségrégés aux joints carbure-carbure a été envisagée. N.K. Sharma et al (1) ont suggéré la présence d'un film de cobalt de $2 \mathrm{~nm}$ entourant les grains de carbure. Cependant les résultats obtenus plus récemment sur la description des joints WC-WC selon le modèle des coincidences géométriques (2) et au cours de la déformation à froid (3) ont reposé le problème de 1 'existence de ce film et donc du squelette continu ou quasi-continu des grains de carbure (4). Aussi nous sommes-nous proposês d'analyser plus précisement la structure intergranulaire des grains WC-WC en comparant les joints de coîncidence et les joints généraux au moyen de la microanalyse $X$.

\section{METHODE EXPERIMENTALE}

Les échantillons de ćarbure examinés contiennent $20 \%$ en poids de cobalt et le diamètre moyen des grains de carbure est voisin du micron. Ils ont été amincis par bombardement ionique (5) $\left(\mathrm{Ar}^{+}, 6 \mathrm{keV}\right)$, sous incidence rasante, pour observation en microscopie êlectronique en transmission. Nous avons successivement effectué sur les joints carbure-carbure une analyse géométrique par diffraction électronique puis une analyse de concentration par microanalyse $\mathrm{X}$.

2.1. Analyse géométrique des joints WC-WC

Par la technique classique de la diffraction electronique en transmission, Ies joints ont été analysés selon la notion des réseaux de coinncidence. I1 est ainsi possible d'attribuer à chacun de ces joints les éléments de la rotation (matrice, 
axe et angle) caractéristiques de la désorientation entre les deux grains contigus. La connaissance de ces éléments permet d'attribuer à chaque joint un indice de coinncidence $\Sigma$ qui est $l^{\prime}$ inverse du nombre de noeuds en coincidence entre les deux grains. Lå liste de ces joints spéciaux a été calculée récemment pour WC qui. a une structure hexagonale avec un rapport $c / a=0,97(2)$. Les joints définis par un indice de coìncidence $\Sigma>50$ seront appelés joints généraux.

\subsection{Microanalyse $\mathrm{X}$ des joints WC-WC}

La microanalyse $X$ intergranulaire a été réalisée sur un microscope Jeo1 $100 \mathrm{CX}$ en mode balayage en transmission, avec un système d'analyse à sélection d'énergie (diode Si-Li et analyseur multicanal SW 9100 Edax). Nous avons opēré avec une énergie des électrons de $100 \mathrm{keV}$ et en mode ponctuel avec une sonde de $20 \mathrm{~nm}$. Nous avons utilisé les raies $\mathrm{L}_{\alpha}(8,425 \mathrm{keV})$ pour W et $\mathrm{K}_{\alpha}(7,065 \mathrm{keV})$ pour Co afin de minimiser les effets d'absorption. Les rapports de concentration $\mathrm{Co} / \mathrm{W}$ ont été déterminés avec le programme quantitatif lame mince Edax, après soustraction du bruit de fond, en appliquant les corrections de fluorescence et avec un $\mathrm{K}$ calculé $=0,248(6)$.

\section{RESULTATS}

La fig. 1 montre la structure granulaire de ce matériau.

3.1. Analyse géométrique. Dans un premier temps nous avons effectué une analyse géométrique des joints WC-WC afin de pouvoir identifier les joints spéciaux. Ce matêriau se caractérise en raison de 1a structure hexagonale de la phase carbure dont le rapport c/a est très voisin de 1, par 1'existence de nombreux joints de coinncidence et essentiellement de joints $\Sigma 2$. On trouve également des joints $\Sigma 13$ et $\Sigma 19$. La proportion de joints en colincidence ou présentant une faible désorientation par rapport à 1 a coincidence est de 1 'ordre de $50 \%$.

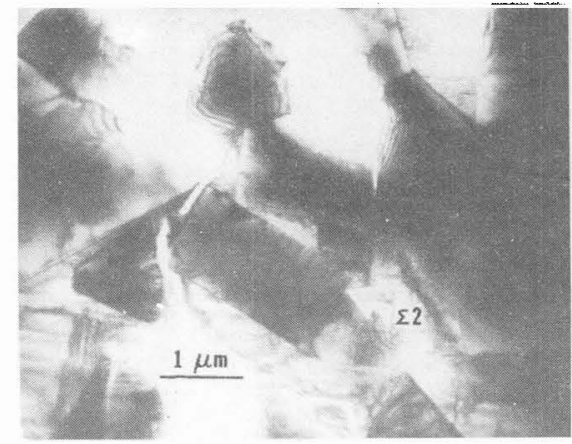

Fig. 1 : Structure granulaire du composite WC-Co $20 \%$

\subsection{Microanalyse $X$ des joints WC-WC}

a) Grains de carbure. - Le tableau 1 montre les rêsultats obtenus sur un échantillonnage de grains. On peut admettre que le rapport $\mathrm{C} / \mathrm{W}$ reste essentie1lement constant à $1^{\prime}$ intérieur d'un grain sauf pour le grain 3 qui se caractérise par un gradient de concentration. Ce rapport se situe entre 0,005 et 0,027. On peut en déduire une concentration intragranulaire moyenne en cobalt qui est de 1 'ordre de $1 \%$ en poids. A partir de ces résultats, il est possible d'analyser la composition des joints et de comparer les joints généraux et les joints de coïncidence.

\begin{tabular}{|c|c|c|c|c|c|c|c|c|c|c|}
\hline grain & 1 & 2 & 3 & 4 & 5 & 6 & 7 & 8 & 9 & 10 \\
\hline$\frac{\text { Co }}{\mathbf{w}}$ & $\begin{array}{l}0,012 \\
0,016\end{array}$ & $\begin{array}{l}0,016 \\
0,014 \\
0,014\end{array}$ & $\begin{array}{l}0,016 \\
0,017 \\
0,021 \\
0,024 \\
0,031\end{array}$ & $\begin{array}{l}0,024 \\
0,027 \\
0,027 \\
0,028 \\
0,028\end{array}$ & $\begin{array}{l}0,006 \\
0,009\end{array}$ & $\begin{array}{l}0,004 \\
0,005\end{array}$ & $\begin{array}{l}0,009 \\
0,010\end{array}$ & 0,005 & 0,006 & 0,010 \\
\hline$\frac{\text { Co }}{w}$ moyen & 0,014 & 0,015 & 0,022 & 0,027 & 0,007 & 0,005 & 0,009 & & & \\
\hline [Co] & 1,3 & 1,4 & 2,1 & 2,5 & 0,7 & 0,5 & 0,8 & 0,5 & 0,6 & 0,9 \\
\hline [Co] moyen & & & & & & $1,1 \%$ & & & & \\
\hline
\end{tabular}

Tableau 1 : Analyse pondêrale intragranulaire (\%) sur les grains de carbure 
b) Joints généraux. - La Fig. 2 montre un exemple de phase cobalt avec trois joints généraux contigus ou 1 'on peut mettre en évidence une forte ségrégation de cobalt. Nous considérerons maintenant le joint général de la Fig. 3 afin de bien montrer les particularités de ce type de microanalyse intergranulaire. La Fig. 4 montre les analyses effectuees en sonde fixe et le tableau 2 donne les résultats correspondants. Il est tout d'abord intéressant de noter que la concentration en tungstène dans la phase cobalt peut être considérëe comme constante $(9,3 \%)$. Le rapport $\mathrm{Co} / \mathrm{W}$ moyen au joint est de $1^{\prime}$ ordre de 0,050 , c'est à dire 5 fois plus élevé que dans les grains (cf spectres, Fig. 5). Les analyses 3 et 5 correspondent manifestement à des pointés sur les grains de carbure, ce qui montre que l'utilisation d'une sonde plus fine (2 nm) n'est pas nécessairement souhaitable ici, car la localisation sur le joint devient extrêmement hasardeuse.

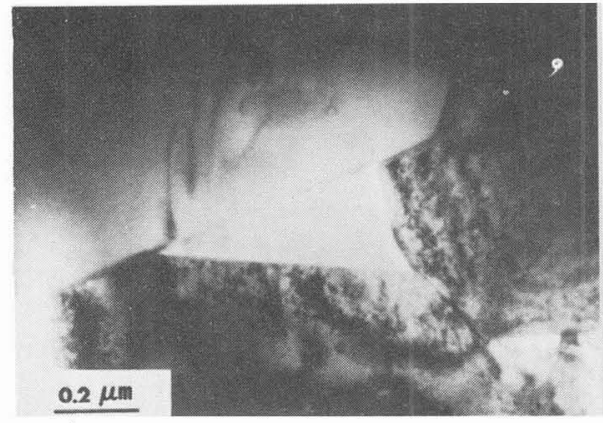

Fig. 2 : WC-Co $20 \%$ phase cobalt

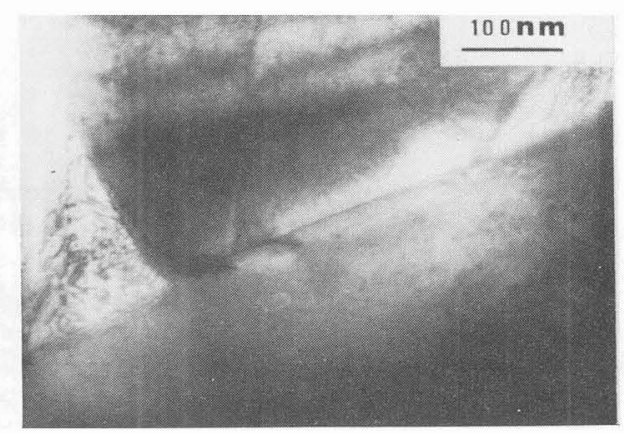

Fig. 3 : Joint général

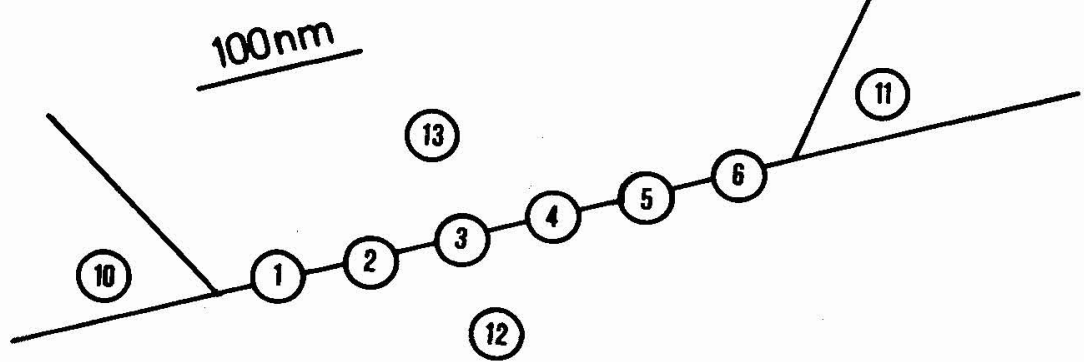

Fig. 4 : Schéma de microanalyse sur le joint général de la Fig. 3

\begin{tabular}{|c|c|c|c|c|c|c|c|c|c|c|}
\hline Spectre & 10 & 1 & 2 & 3 & 4 & 5 & 6 & 11 & 12 & 13 \\
\hline$\frac{\mathrm{Co}}{W}\left\{\begin{array}{l}\mathrm{Co}_{0} \\
\mathrm{~W}_{0}^{\circ}\end{array}\right.$ & $\begin{array}{r}90,5 \\
9,5\end{array}$ & $\begin{array}{r}4,7 \\
95,3\end{array}$ & $\begin{array}{r}4,9 \\
95,1\end{array}$ & & $\begin{array}{r}5,1 \\
94,9\end{array}$ & & $\begin{array}{r}4,1 \\
95,9\end{array}$ & $\begin{array}{r}90,6 \\
9,4\end{array}$ & $\begin{array}{r}0,6 \\
99,4\end{array}$ & $9^{1,0}$ \\
\hline$\frac{C_{0}}{W}$ & 38,2 & 0,050 & 0,052 & 0,014 & 0,054 & 0.009 & 0,043 & 38,1 & 0,006 & 0,010 \\
\hline$\left[\begin{array}{l}\mathrm{Co} \\
\mathrm{WC}\end{array}\right]$ & $\begin{array}{r}90,6 \\
9,4\end{array}$ & $\begin{array}{r}4,4 \\
95,6\end{array}$ & $\begin{array}{r}4,6 \\
95,4\end{array}$ & & $\begin{array}{r}4,8 \\
95,2\end{array}$ & & $\begin{array}{r}3,9 \\
96,1\end{array}$ & $\begin{array}{r}90,7 \\
9,3\end{array}$ & $\begin{array}{r}0,6 \\
99,4\end{array}$ & $\begin{array}{r}0,9 \\
99,1\end{array}$ \\
\hline [Co] moyen & & & & 4,4 & & & & & & \\
\hline
\end{tabular}

Tableau 2 : Analyse pondérale intergranulaire (\%) sur un joint gênêral (Fig. 3) 

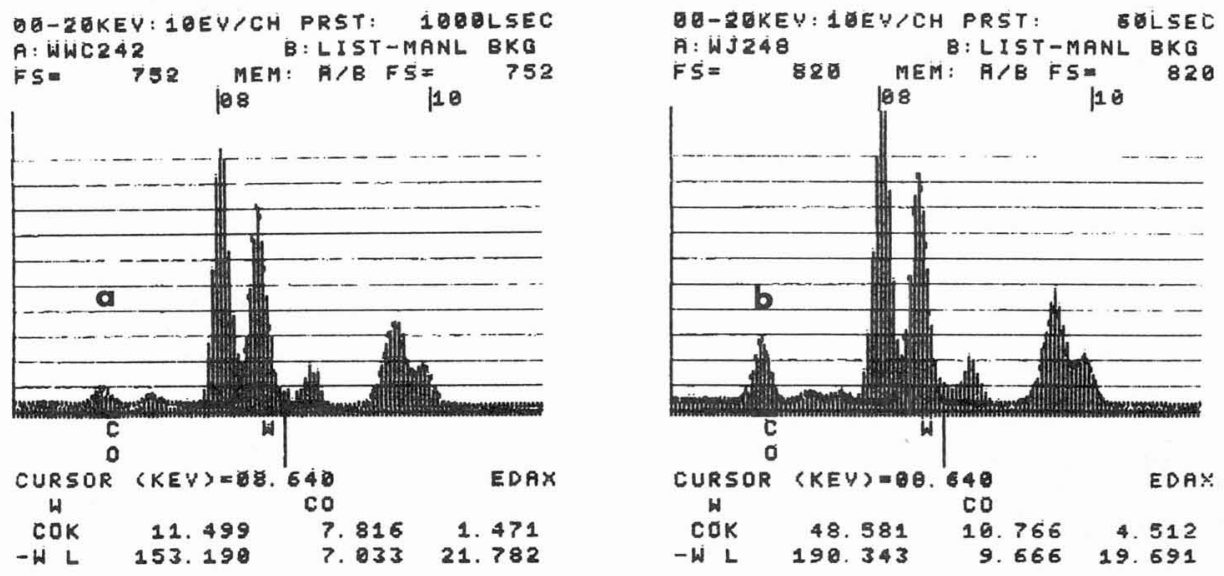

Fig. 5 : Spectres X (sélection d'énergie)

5a) grain WC $5 b$ ) joint général contigu (Fig. 4)

c) Joints de coincidence. - Considérons maintenant le cas des joints de coinncidence. La Fig. 6 montre les pointés effectués sur les joints $\Sigma 2$ de la Fig. 1 et de part et d'autre. Le tableau 3 et la Fig. 7 montrent les résultats correspondants. On voit que la concentration en cobalt dans les grains contigus est relativement élevée et que 1 'un des grains se caractérise par un gradient de concentration croissant vers le joint, ce qui serait a priori plus favorable que le cas précédent (tableau 2) pour obtenir une ségrégation intergranulaire de cobalt. Le pointé 1 effectué à $150 \mathrm{~nm}$ du point triple montre effectivement un excès de cobalt dû à la phase cobalt voisine. Par contre, toutes les autres analyses donnent des résultats qui correspondent aux valeurs intergranulaires. Compte tenu des conditions opératoires et si l'on se rapporte à l'exemple précédent, 1 'hypothèse suivant laquelle les analyses 2 à 7 se situeraient hors joint peut être exclue. I1 est donc possible d'affirmer qu'il n'y a pas de ségrégation de cobalt sur ce type de joint, ou tout au moins qu'elle est parfaitement nëgligeable par rapport au cas des joints généraux.

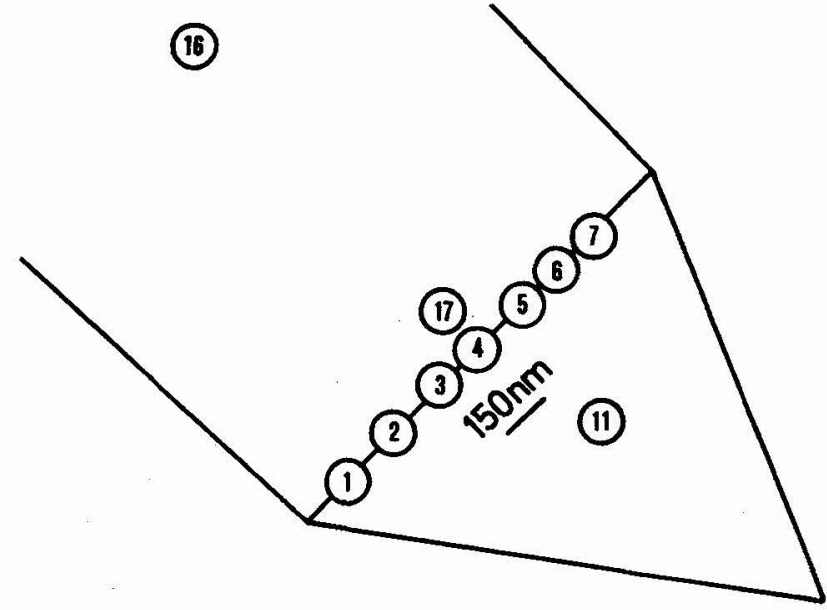

Fig. 6 : Schëma de microanalyse sur le joint $\Sigma 2$ de 1a Fig. 1 


\begin{tabular}{|c|c|c|c|c|c|c|c|c|c|}
\hline Spectre & 1 & 2 & 3 & 4 & 5 & 6 & 7 & 11 & 12 à 17 \\
\hline$\frac{C_{0}}{W}\left\{\begin{array}{l}C_{0} \\
W\end{array}\right.$ & $\begin{array}{r}5,3 \\
94,7\end{array}$ & $\begin{array}{r}3,0 \\
97,0\end{array}$ & $\begin{array}{r}2,9 \\
97,1\end{array}$ & $\begin{array}{r}2,5 \\
97,5\end{array}$ & $\begin{array}{r}2,5 \\
97,5\end{array}$ & $\begin{array}{r}2,2 \\
97,8\end{array}$ & $\begin{array}{r}2,6 \\
97,4\end{array}$ & & \\
\hline$\frac{\mathrm{Co}}{\mathrm{w}}$ & 0,056 & 0,031 & 0,030 & 0,026 & 0,025 & 0,022 & 0,027 & 0,027 & $\begin{array}{c}0,016 \\
\text { to } 0,031\end{array}$ \\
\hline$\left[\begin{array}{l}\mathrm{Co} \\
\mathrm{w}]\end{array}\right]$ & ${ }_{95}^{5,0}$ & $\begin{array}{r}2,8 \\
97,2\end{array}$ & $\begin{array}{r}2,7 \\
97,3\end{array}$ & $\begin{array}{r}2,4 \\
97,6\end{array}$ & $\begin{array}{r}2,4 \\
97,6\end{array}$ & $\begin{array}{r}2,1 \\
97,9\end{array}$ & $\begin{array}{r}2,5 \\
97,5\end{array}$ & $\begin{array}{r}2,5 \\
97,5\end{array}$ & $\begin{array}{r}2,1 \\
97,9\end{array}$ \\
\hline [Co] moyen & & & & 2,5 & & & & & \\
\hline
\end{tabular}

Tableau 3 : Analyse pondérale intergranulaire (\%) sur un joint $\sum 2$ (Fig.1)
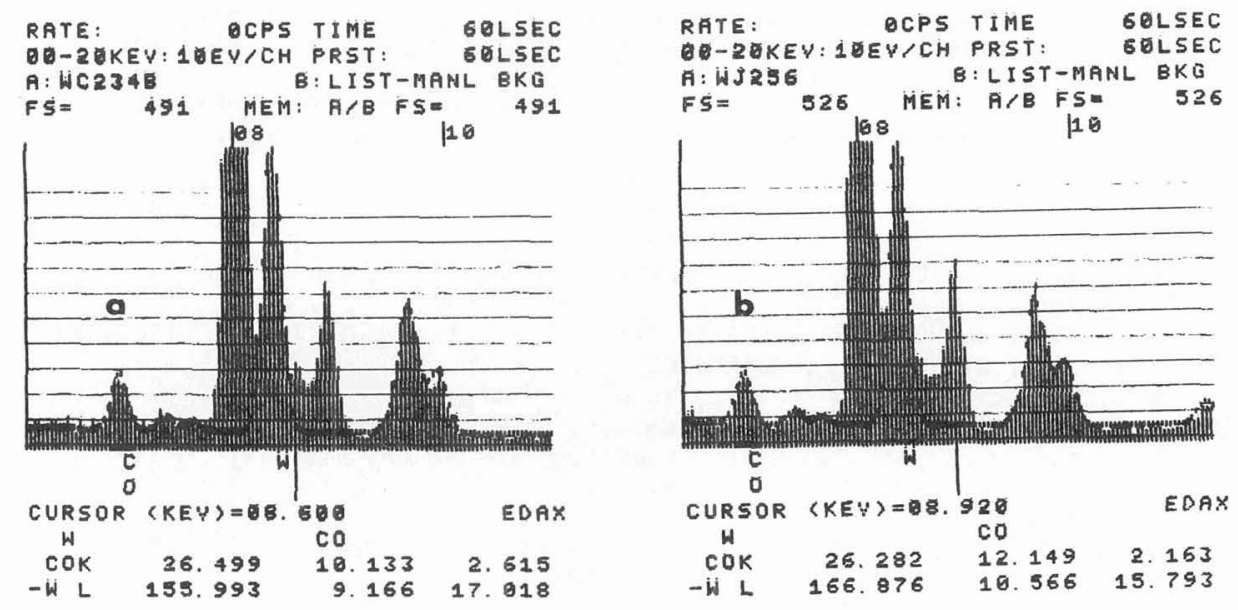

Fig. 7 : Spectres $X$ (sélection d'énergie)

5a) grain WC 5b) joint $\Sigma 2$ contigu (Fig.1)

\section{INTERPRETATION}

Ces résultats comparatifs sont particulièrement intéressants si on les rapporte aux tentatives de visualisation d'une phase ou d'un film cobalt au joint de carbure. Ils vont en effet nous permettre de conclure à coup sûr quant à la structure des joints spêciaux. En effet, si aucune phase cobalt n'a pu être mise en évidence jusqu'à présent dans le cas des joints généraux bien que le rapport de concentration $\mathrm{Co} / \mathrm{W}$ augmente dans un rapport 5 au niveau du joint et si 1 'on se souvient qu'il est possible de visualiser des films aussi fins que $2 \mathrm{~nm}$ en microscopie êlectronique haute résolution (7) ou par la technique des effets induits (8), il est clair qu'il ne peut exister de film de cobalt sur les joints de coincidence où le rapport $\mathrm{Co} / \mathrm{W}$ ne varie pas.

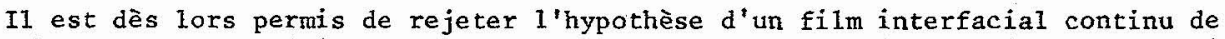
cobalt sur ces matériaux. La microstructure intergranulaire au niveau des grains de carbure dépendra alors de la concentration en cobalt du composite. Pour de faible concentration en cobalt, on aura percolation à travers les joints spéciaux et on obtiendra un squelette continu de carbure. Pour des concentrations moyennes et plus élevées (jusqu'à $20 \%$ ) il ne sera plus possible d'obtenir un 
squelette continu, mais on obtiendra nêanmoins dés grappes à l'intérieur desquelles on aura toujours un squelette carbure continu. L'évolution des propriétés mécaniques avec la concentration en cobalt (9) s'interprète bien avec une telle microstructure - alors qu'elle ne peut être expliquêe - en présence d'un film continu de cobalt.

\section{CONCLUSION}

La microanalyse $\mathrm{X}$ en microscopie électronique à transmission en balayage nous a permis de mettre en êvidence une nette différence de composition entre les joints de carbure spéciaux et généraux. Dans le cas des joints généraux, la concentration en cobalt passe de $1 \%$ dans la matrice à $4,5 \%$. Par contre dans le cas des joints spéciaux, on n'observe pas de variation de concentration. Aucun film de cobalt n'ayant pu être mis en évidence dans le cas des joints généraux, on pourra donc affirmer a fortiori qu'il ne peut en aucun cas y avoir de film de cobalt interfacial dans le cas des joints spêciaux. Par consêquent, il existe bien un squelette continu ou quasi-continu de carbure, selon la concentration en cobalt.

Ces résultats montrent que la microanalyse X intergranulaire en S.T.E.M. peut fournir des informations précises à condition de l'appliquer à des joints bien définis. Ainsi dans le cas particulier des composites carbures de tungstène-cobalt, la microanalyse $X$ permet de conclure à l'existence d'un squelette carbure uniquement si l'on $s^{\prime}$ appuie sur les critères prêcis de coincidence cristallographique.

\section{REFERENCES}

(1) N.K. SHARMA, I.D. WARD, H.L. FRASER, W.S. WLLLIAMS, Am. Ceram. Soc. 63 (1980) 194

(2) S. HAGEGE, G. NOUET, P. DELAVIGNETTE, Phys. Stat. Sol. (a) 61 (1980) 97

(3) S. HAGEGE, G. NOUET, F. OSTERSTOCK, J. VICENS, J.L. CHERMANT Mem. Et. Scient. Revue de Métallurgie 9 (1981) 404

(4) S. HAGEGE, J. VICENS, P. DELAVIGNETTE, G. NOUET, Proceedings of the 2nd European Conf. on Solid State Chemistry, Veldhoven, E1sevier, 3 (1983) 447

(5) M. PAULUS, F. REVERCHON, Le bombardement ionique, Colloque Int. CNRS $\mathrm{N}^{\circ} 113-223(1961)$

(6) N.J. ZALUJEC, Quantitative X-ray analysis, Plenum Press (1979) 121

(7) C. GRESROVICH, J. Mat. Science 11 (1976) 1135

(8) J.Y. LAVAL, M.C. AMAMRA, D. BROUSSAUD, W. MUSTEL, Rev. Chimie Minérale 19 (1982) 690

(9) J.G. BALDONI, W.S. WILliaMS, American Ceramic Society Bulletin, 57 (1978) 1100

*Les travaux très récents de Jayaram et al en microscopie électronique haute résolution (J. Am. Ceram. Soc., 66 (1983) C-137) confirment bien d'une part, la présence de cobalt sur des joints généraux et d'autre part, I'absence de film de cobalt ou de déformation réticulaire locale dans un joint $\Sigma 2$. Ces auteurs montrent ainsi pour la première fois $1 \mathrm{a}$ présence d'un film nanométrique au niveau d'un joint gënéral. Ils montrent également 1a_présence d'un film de co sur un autre joint qui se caractérise par des plans (1īoo) parallèles dans les deux grains adjacents : Il convient de noter qu'une telle orientation équivaut à une rotation quelconque autour de $I^{\prime}$ axe (1100) et ne correspondra qu'exceptionnellement à une orientation de coìncidence. 